\title{
Global Existence and Asymptotics of the Solutions of the Second-Order Quasilinear Hyperbolic Equations with the First-Order Dissipation
}

\author{
By
}

\author{
Akitaka MAtsumura*
}

\section{Introduction}

In this paper, we first consider the following Cauchy problem for the quasilinear hyperbolic equations

$$
\begin{aligned}
& L(u)=u_{t t}-\sum_{i, j=1}^{n} a_{i j}(x, t, D u) u_{i j}+\alpha u_{t}+b(D u)=0 \\
& \left\{\begin{array}{l}
u(x, 0)=\phi(x) \\
u_{t}(x, 0)=\psi(x)
\end{array}\right.
\end{aligned}
$$

where $x \in \mathbb{R}^{n}, t \geq 0, \alpha>0, u_{i}=\frac{\partial u}{\partial x_{i}}, u_{t}=\frac{\partial u}{\partial t}$ and

$$
D u=\left(u, u_{t}, u_{1}, u_{2}, \ldots, u_{n}\right)
$$

Here the coefficients $a_{i j}$ are smooth and satisfy

$$
\sum_{i, j} a_{i j}(x, t, y) \check{\zeta}_{i} \xi_{j} \geq a(y) \sum_{i} \xi_{i}^{2}, \quad a(0)>0
$$

for all $x \in \mathbb{R}^{n}, t \in \boldsymbol{R}^{1}, y \in \mathbb{R}^{n+2}, \xi \in \mathbb{R}^{n}$.

Recently, we investigated the global existence and decay of the solutions of the semilinear wave equations

$$
u_{t t}-\Delta u+\alpha u_{t}+b(D u)=0 \quad x \in \mathbb{R}^{n}, \quad t \geq 0, \quad \alpha>0
$$

Communicated by S. Matsuura, June 24, 1976.

* Department of Applied Mathematics and Physics, Faculty of Engineering, Kyoto University, Kyoto 606, Japan. 
with the small data in [2]. For the space dimension $n=1$, Nishida [6] showed that the quasilinear equations

$$
u_{t t}-\frac{\partial}{\partial x} \sigma\left(u_{x}\right)+\alpha u_{t}=0 \quad x \in \boldsymbol{R}^{n}, \quad t \geq 0, \quad \alpha>0
$$

have the global smooth solutions for the small data. But his argument is not applicable to the cases $n \geq 2$. In $\S 2$ we establish the global existence and decay theorem of (1) for general cases $n \geq 1$ with small data and boundedness of some coefficients (Theorem 2).

Next we consider the following initial-boundary value problem;

$$
\begin{aligned}
& L(u)=\varepsilon f(x, t) \quad x \in \Omega, \quad t \geq 0 \\
& \left\{\begin{array}{l}
u(x, 0)=\phi(x) \\
u_{t}(x, 0)=\psi(x) \\
\left.u\right|_{\partial \Omega}=0
\end{array}\right.
\end{aligned}
$$

where $\Omega$ is a bounded open set in $R^{n}$ with smooth boundary $\partial \Omega$ and $\varepsilon$ is a sufficiently small constant. For the semilinear equations

$$
u_{t t}-\sum_{i, j} a_{i j}(x) u_{i j}+\alpha u_{t}=b(u) \quad x \in \Omega, \quad t \geq 0, \quad \alpha>0,
$$

Sattinger [7] discussed the global existence and stability with small data. In $\S 3$ we establish the global existence and decay theorem (Theorem 3) even for general quasilinear equations (1)' under the assumptions stated in Theorem 3.

Moreover, at the end of $\S 3$, we mention the results of the existence, uniqueness and stability of the time periodic solutions for

$$
\begin{aligned}
& L(u)=\varepsilon f(x, t) \quad x \in \Omega, \quad t \in \boldsymbol{R}^{1} \\
& \left.u\right|_{\partial \Omega}=0
\end{aligned}
$$

where $\Omega$ is a bounded open set in $\boldsymbol{R}^{n}$ with smooth boundary $\partial \Omega$ and $\varepsilon$ is a sufficiently small constant. For the semilinear equations

$$
u_{t t}-\sum_{i, j} a_{i j}(x) u_{i j}+\alpha u_{t}=\varepsilon f(x, t, D u) \quad x \in \Omega, t \in \boldsymbol{R}^{1}, \alpha>0,
$$


Rabinowitz [8] showed existence and stability of the time periodic solutions. Moreover he [9] showed existence only for the nonlinear equations

$$
\begin{aligned}
& u_{t t}-u_{x x}+\alpha u_{t}=\varepsilon f\left(x, t, D u, u_{t t}, u_{t x}, u_{x x}\right) \\
& x \in(a, b) \subset \boldsymbol{R}^{1}, \quad t \in \boldsymbol{R}^{1}, \quad \alpha>0 .
\end{aligned}
$$

Although our (1)" are quasilinear, we can establish not only existence for more general space dimension $n \geq 1$ but also stability (Corollary of Theorem 3).

\section{Notations and Preliminaries}

In this paper, all functions are real valued. Let $\Omega$ be $\mathbb{R}^{n}$ or a bounded open set in $\boldsymbol{R}^{n}$ with the $C^{\infty}$-boundary $\partial \Omega$. We denote by $L^{p}(\Omega)(1 \leq p \leq \infty)$ the space of measurable functions $u$ on $\Omega$ whose $p$-th powers are integrable with the norm

$$
\|u\|_{p}=\left(\int|u(x)|^{p} d x\right)^{\frac{1}{p}}, \quad\|u\|_{\infty}=\underset{x \in \Omega}{\operatorname{ess.sup}}|u(x)| .
$$

If $p=2$, we write $\|\cdot\|$. Let $f(z)$ be a function of $z \in \mathbb{R}^{r}$ ( $r$ is some positive integer). Then $D_{z}^{k} f$ (resp. $\bar{D}_{z}^{k} f$ ) ( $k$ is some positive integer) represents the vector which has

$$
\frac{(k+r) !}{k ! r !} \quad\left(\text { resp. } \frac{(k+r) !}{k ! r !}-1\right)
$$

components,

$$
\begin{aligned}
D_{z}^{k} f=\left\{\left(\frac{\partial}{\partial z}\right)^{\alpha} f\right\}, & 0 \leq|\alpha| \leq k \\
\text { (resp. } \bar{D}_{z}^{k} f=\left\{\left(\frac{\partial}{\partial z}\right)^{\alpha} f\right\}, & 1 \leq|\alpha| \leq k)
\end{aligned}
$$

where $\alpha=\left(\alpha_{1}, \alpha_{2}, \ldots, \alpha_{r}\right)$ and $|\alpha|=\sum_{i=1}^{r} \alpha_{i}$.

Especially, $D^{k} f$ and $D^{k, m} f$ represent

$$
D^{k} f=\left\{\left(\frac{\partial}{\partial x}\right)^{\alpha}\left(\frac{\partial}{\partial t}\right)^{i} f\right\}, \quad 0 \leq i+|\alpha| \leq k,
$$




$$
D^{k, m} f=\left\{\left(\frac{\partial}{\partial x}\right)^{\alpha}\left(\frac{\partial}{\partial t}\right)^{i} f\right\}, \quad 0 \leq|\alpha| \leq k, 0 \leq i \leq m .
$$

$\bar{D}^{k} f$ and $\bar{D}^{k, m} f$ are similarly defined as $\bar{D}_{z}^{k} f$. If $k=1$ for $D_{z}^{k} f$, we write simply $D_{z} f$. Moreover $D_{z}^{k} f \cdot D_{z}^{k} g$ (resp. $\bar{D}_{z}^{k} f \cdot \bar{D}_{z}^{k} g$ ) represents the usual vector inner product for $D_{z}^{k} f$ and $D_{z}^{k} g$ (resp. $\bar{D}_{z}^{k} f$ and $\bar{D}_{z}^{k} g$ ).

For some set $G$ in $R^{r}, C^{k}(G)$ is the space of the real valued functions on $G$ that are $k$-times continuously differentiable. $C_{0}^{\infty}(\Omega)$ denotes the space of $C^{\infty}(\Omega)$ functions with compact support in $\Omega . H^{k}$ denotes the space of functions all of whose derivatives of order $\leq k$ belong to $L^{2}(\Omega)$ and the norm of $H^{k}$ is equal to $\left\|D_{x}^{k} \cdot\right\|$. The completion of the space of $C_{0}^{\infty}(\Omega)$ functions by the $H^{k}$ norm is denoted by $H^{k}$.

Let $X$ be a Banach space on $\Omega$. Then $u(x, t) \in \mathscr{E}_{t}^{k}(X)$ (resp. $L_{t}^{\infty}(X)$ ) $\left(t_{0} \leq t \leq t_{1}\right)$ means that $u(\cdot, t)$ belongs to $X$ for every fixed $t$ and $u$ is $k$-times continuously differentiable (resp. bounded) with respect to $t$ in $X$-topology on $t_{0} \leq t \leq t_{1}$.

We use $c_{i}$ as the constants, especially use $c$ for the constants which we need not distinguish and write $c_{i}(X)$ when we emphasize its dependence on $X$. We denote by $h_{i}(\tau)$ the continuous nonnegative and nondecreasing functions on $\tau \geq 0$.

We note the next Sobolev's inequalities.

Lemma 1 (Mizohata [3] Chapter 7). We suppose $\Omega$ is as in the above.

i) If $u \in H^{\left[\frac{n}{2}\right]+1+m}(m \geq 0)$, we have

$$
\left\|D_{x}^{m} u\right\|_{\infty} \leq c_{0}(m, n, \Omega)\left\|D_{x}^{\left[\frac{n}{2}\right]+1+m} u\right\|
$$

ii) If $u \in H^{\left[\frac{n}{2}\right]+1+m}(m \geq 0)$, we have for $m+1 \leq|\alpha| \leq m+1+\left[\frac{n}{2}\right]$

$$
\left(\frac{\partial}{\partial x}\right)^{\alpha} u \in L^{p}
$$

and

$$
\left\|\left(\frac{\partial}{\partial x}\right)^{\alpha} u\right\|_{p} \leq c_{0}(p, m, n, \alpha, \Omega)\left\|D_{x}^{\left[\frac{n}{2}\right]+1+m} u\right\|
$$


where

$$
\begin{cases}\frac{1}{p} \in\left[\frac{|\alpha|}{n}-\frac{m+1}{n}, \frac{1}{2}\right]-0 & (n=\text { even }) \\ \frac{1}{p} \in\left[\frac{|\alpha|}{n}-\frac{2 m+1}{n}, \frac{1}{2}\right] & (n=\text { odd }) .\end{cases}
$$

\section{$\S 1$. Basic Estimates}

In this section we show the estimates of $a_{i j}(x, t, D u)$ and $b(D u)$. We list up the following assumptions $\left(s=\left[\frac{n}{2}\right]+2\right)$.

\section{Assumption 1.}

i) $a_{i j}(x, t, y) \in C^{s+1}\left(\boldsymbol{R}^{n} \times \boldsymbol{R}^{1} \times \boldsymbol{R}^{n+2}\right) \quad$ for $1 \leq i, j \leq n$.

ii) $a_{i j}=a_{j i} \quad$ for $1 \leq i, j \leq n$.

iii) $\sum_{i, j} a_{i j}(x, t, y) \xi_{i} \xi_{j} \geq a(y) \sum_{i} \xi_{i}^{2}, \quad a(0)=a_{0}>0$

for all $x \in \boldsymbol{R}^{n}, t \in \boldsymbol{R}^{1}, y \in \boldsymbol{R}^{n+2}, \xi \in \boldsymbol{R}^{n}$

where $a(y) \in C^{0}\left(\boldsymbol{R}^{n+2}\right)$.

iv) $\sup _{\boldsymbol{R}^{n} \times \mathbb{R}^{1}} \sum_{i, j}\left|D_{x, t, y}^{s+1} a_{i j}(x, t, y)\right| \leq h_{0}(|y|)$.

\section{Assumption 2.}

$$
\sup _{\boldsymbol{R}^{n} \times \boldsymbol{R}^{1}} \sum_{i, j}\left|\bar{D}_{x} a_{i j}(x, t, y)\right| \leq|\tilde{y}| h_{0}(|y|) \quad \text { for } \quad|\tilde{y}| \leq 1
$$

where $\tilde{y}=\left(0, y_{2}, y_{3}, \ldots, y_{n+2}\right)$.

\section{Assumption 3.}

i) $b(y) \in C^{s+1}\left(\boldsymbol{R}^{n+2}\right), \quad\left|D_{y}^{s+1} b(y)\right| \leq h_{1}(|y|)$.

ii) $D_{y} b(0)=0$.

\section{Assumption 4.}

i) $b(D u)=b_{1}(u)+b_{2}(D u)$. 
ii) $b_{1}(u) u \geq 0$.

iii) $b_{2}(y) \leq|\tilde{y}|^{2} h_{1}(|y|)$ for $|\tilde{y}| \leq 1$.

Remark. Throughout this paper $s$ represents $\left[\frac{n}{2}\right]+2$ and $|\cdot|$ denotes the usual Euclidian norm.

By Assumption 1, we can choose the positive constants $\gamma_{0}$ and $a_{1}$ $\left(0<a_{1}<a_{0}\right)$ such that

$$
a(y)>a_{1}>0 \text { if }|y| \leq \gamma_{0} .
$$

Moreover, by Lemma 1, we can choose a positive constant $e_{0}$ such that

$$
\sup _{0 \leq t \leq T}\|D u(t)\|_{\infty} \leq \gamma_{0} \quad \text { if } \sup _{0 \leq t \leq T}\left\|D^{s} u(t)\right\| \leq e_{0}
$$

where $T$ is any positive constant. So we define the space of $u(x, t)$, $\mathscr{D}(0, T \mid e)$, for $0<e \leq e_{0}$ by

$$
\begin{array}{r}
\mathscr{D}(0, T \mid e)=\left\{u(x, t) \mid D^{s+1} u(x, t) \in \mathscr{E}_{t}^{\circ}\left(L^{2}\right)(0 \leq t \leq T) \quad\right. \text { and } \\
\left.\sup _{0 \leq t \leq T}\left\|D^{s+1} u(t)\right\| \leq e \quad\left(0<e \leq e_{0}\right)\right\} .
\end{array}
$$

Now we define $E_{v}\{u(t)\}$ by

$$
\begin{aligned}
E_{v}\{u(t)\}=\int & \frac{\lambda}{2}\left|D^{s} u\right|^{2}+\lambda D^{s} u \cdot D^{s} u_{t}+\frac{1}{2}\left|D^{s} u_{t}\right|^{2} \\
& +\frac{1}{2} \sum a_{i j}(x, t, D v) D^{s} u_{i} \cdot D^{s} u_{j} d x \quad(0<\lambda<1) .
\end{aligned}
$$

Then we note the following under Assumption 1.

Lemma 2. If $v \in \mathscr{D}(0, T \mid e), E_{v}\{u(t)\}$ is equivalent to $\left\|D^{s+1} u(t)\right\|^{2}$ for $0 \leq t \leq T$, that is,

$$
c_{1}\left\|D^{s+1} u(t)\right\|^{2} \leq E_{v}\{u(t)\} \leq c_{2}\left\|D^{s+1} u(t)\right\|^{2} \quad \text { for } \quad 0 \leq t \leq T,
$$

where $c_{1}$ and $c_{2}$ depend only on $e_{0}, c_{0}, a_{1}, h_{0}$ and $\lambda_{\text {. }}$

This lemma is easily verified by Assumption 1 and (4) (6).

In order to estimate $\sum a_{i j} u_{i j}$ and $b$, we note the following estimates 
of the composite functions.

\section{Lemma 3.}

i) Suppose that

$$
\left\{\begin{array}{l}
f(x, t, y) \in C^{s+1}\left(\boldsymbol{R}^{n} \times \boldsymbol{R}^{1} \times \boldsymbol{R}^{n+2}\right) \\
\sup _{\mathbb{R}^{n} \times \boldsymbol{R}^{1}}\left|D_{x, t, y}^{s+1} f(x, t, y)\right| \leq h_{2}(|y|) \\
v(x, t) \in \mathscr{E}_{t}^{i}\left(H^{s+1-i}\right)(0 \leq i \leq s+1), \quad w(x, t) \in \mathscr{E}_{t}^{i}\left(H^{s-1-i}\right) \\
(0 \leq i \leq s-1) .
\end{array}\right.
$$

Then it follows for $1 \leq k \leq s$ that

$$
\left\|D^{k}\{f(x, t, D v(x, t)) w(x, t)\}-f(x, t, D v(x, t)) D^{k} w(x, t)\right\|
$$

$$
\begin{aligned}
& \leq c\left\{f_{0}+\left(\left\|D^{s+1} v\right\|+\left\|D^{s+1} v\right\|^{s}\right) h_{2}\left(\|D v\|_{\infty}\right)\right\}\left\|D^{k-1} w\right\| \\
& \leq c\left(1+\left\|D^{s+1} v\right\|^{s}\right)\left\|D^{s-1} w\right\| h_{2}\left(\|D v\|_{\infty}\right)
\end{aligned}
$$

where

$$
f_{0}=\sup _{\boldsymbol{R}^{n} \times \boldsymbol{R}^{1}}\left|\bar{D}^{s} f(x, t, 0)\right|
$$

ii) Suppose that

$$
\left\{\begin{array}{l}
g(y) \in C^{s+1}\left(\boldsymbol{R}^{n+2}\right), \quad g(0)=0 \\
\left|D_{y}^{s+1} g(y)\right| \leq h_{3}(|y|) \\
v(x, t) \in \mathscr{E}_{t}^{i}\left(H^{s+1-i}\right) \quad(0 \leq i \leq s+1) .
\end{array}\right.
$$

Then it follows for $0 \leq k \leq s$ that

$$
\left\|D^{k} g(D v(x, t))\right\|
$$

$$
\begin{aligned}
& \leq c\left\{g_{0}+\left(\left\|D^{s+1} v\right\|+\left\|D^{s+1} v\right\|^{s-1}\right) h_{3}\left(\|D v\|_{\infty}\right)\right\}\left\|D^{k+1} v\right\| \\
& \leq c\left(\left\|D^{s+1} v\right\|+\left\|D^{s+1} v\right\|^{s}\right) h_{3}\left(\|D v\|_{\infty}\right)
\end{aligned}
$$

where

$$
g_{0}=\left|D_{y} g(0)\right|
$$


Remark. We can get (7) (8)' by using Lemma 1 and especially Taylor's formula for (7), (7)' (refer to Chapter I and II in Dionne [1]). In this paper, we use more precise forms (7), (7)' rather than (8), (8)'.

Now, defining $\varepsilon_{1}$ by

$$
\varepsilon_{1}=\sup _{\boldsymbol{R}^{n} \times \boldsymbol{R}^{1}} \sum\left|\bar{D}^{s} a_{i j}(x, t, 0)\right|,
$$

we have the following (we omit $\Sigma$ for simplicity)

Lemma 4. Suppose Assumption 1, then for $u(x, t)$ and $v(x, t) \in$ $\mathscr{E}_{t}^{i}\left(H^{s+1-i}\right)(0 \leq i \leq s+1)$ we have the following:

i) $\left\|D^{k}\left(a_{i j} u_{i j}\right)-a_{i j} D^{k} u_{i j}\right\|$

$$
\leq c\left(\left\|D^{s+1} v\right\| h_{0}+\left\|D^{s+1} v\right\|^{s} h_{0}+\varepsilon_{1}\right)\left\|\bar{D}^{k+1} u\right\|
$$

where $a_{i j}=a_{i j}(x, t, D v), h_{0}=h_{0}\left(\|D v\|_{\infty}\right), 1 \leq k \leq s$.

ii) $\left\|\left(a_{i j} D^{k} u_{i} \cdot D^{k} u_{t}\right)_{j}-a_{i j} D^{k} u_{i j} \cdot D^{k} u_{t}-a_{i j} D^{k} u_{i} \cdot D^{k} u_{t j}\right\|_{1}$

$$
\begin{aligned}
& +\left\|\frac{1}{2}\left(a_{i j} D^{k} u_{i} \cdot D^{k} u_{j}\right)_{t}-a_{i j} D^{k} u_{i} \cdot D^{k} u_{j t}\right\|_{1} \\
& \leq c\left(\left\|D^{s+1} v\right\| h_{0}+\varepsilon_{1}\right)\left\|\bar{D}^{k+1} u\right\|^{2} \quad 1 \leq k \leq s .
\end{aligned}
$$

iii) $\left\|\left(a_{i j} D^{k} u_{i} \cdot D^{k} u\right)_{j}-a_{i j} D^{k} u_{i j} \cdot D^{k} u-a_{i j} D^{k} u_{i} \cdot D^{k} u_{j}\right\|_{1}$

$$
\leq c\left(\left\|D^{s+1} v\right\| h_{0}+\varepsilon_{1}\right)\left\|D^{k+1} u\right\|^{2} \quad 1 \leq k \leq s .
$$

iii)' Suppose in addition Assumption 2. Then, left hand side of iii) $\leq c\left\|\bar{D}^{s+1} v\right\|\left\|\bar{D}^{s+1} u\right\|\left\|D^{s+1} u\right\| h_{0}$.

iv) $\left\|D^{k}\left\{a_{i j}(D u)-a_{i j}(D v)\right\} u_{i j}\right\|$

$$
\begin{aligned}
& \leq c\left\|D^{k+1}(u-v)\right\|\left\|D^{s+1} u\right\|\left(1+\left\|D^{s+1} u\right\|^{s-2}+\left\|D^{s+1} v\right\|^{s-2}\right) \\
& \quad \times h_{0}\left(\|D u\|_{\infty}+\|D v\|_{\infty}\right) \quad 0 \leq k \leq s-1 .
\end{aligned}
$$

v) Suppose in addition $v \in \mathscr{D}(0, T \mid e)$. Then,

$$
\left\|D_{x}^{2} u\right\| \leq c\left(e_{0}, h_{0}, a_{1}, \Omega\right)\left\{\left\|a_{i j}(D v) u_{i j}\right\|+\left\|D_{x}^{1} u\right\|\right\} \quad \text { for } \quad 0 \leq t \leq T .
$$

Lemma 5. Suppose Assumption 3, then for $u(x, t)$ and $v(x, t)$ 
$\in \mathscr{E}_{t}^{i}\left(H^{s+1-i}\right)(0 \leq i \leq s+1)$ we have the following:

i) $\left\|D^{k} b(D u)\right\| \leq c\left\|D^{k+1} u\right\|\left(\left\|D^{s+1} u\right\|+\left\|D^{s+1} u\right\|^{s-1}\right) h_{1}\left(\|D u\|_{\infty}\right) \quad 0 \leq k \leq s$.

ii) $\left\|\bar{D}^{s} b(D u)\right\| \leq c\left\|D^{s+1} u\right\|\left\|\bar{D}^{s+1} u\right\|\left(1+\left\|D^{s+1} u\right\|^{s-2}\right) h_{1}\left(\|D u\|_{\infty}\right)$.

iii) $\left\|D^{k}\{b(D u)-b(D v)\}\right\|$

$$
\begin{aligned}
\leq & c\left\|D^{k+1}(u-v)\right\|\left(\left\|D^{s+1} u\right\|+\left\|D^{s+1} v\right\|\right) \\
& \times\left(1+\left\|D^{s+1} u\right\|^{s-2}+\left\|D^{s+1} v\right\|^{s-2}\right) h_{1}\left(\|D u\|_{\infty}+\|D v\|_{\infty}\right) \quad 0 \leq k \leq s .
\end{aligned}
$$

iv) Suppose in addition Assumption 4. Then,

$$
\left\|b_{2}(D u)\right\| \leq c\|\bar{D} u\|^{2} h_{1}\left(\|D u\|_{\infty}\right)
$$

Remarks. Lemmas 4 and 5 (except v) of Lemma 4) are given by using Lemmas 1 and 3 (refer to the Chapter I and II in Dionne [1]) and v) of Lemma 4 is shown by the strong (uniform) ellipticity of $\sum a_{i j}(D v) u_{i j}$ with $v \in \mathscr{D}(0, T \mid e)$.

\section{§2. Cauchy Problem}

In this section, we consider the Cauchy problem

$$
\begin{aligned}
& L(u)=u_{t t}-\sum_{i, j} a_{i j}(x, t, D u) u_{i j}+\alpha u_{t}+b(D u)=0 \\
& x \in \boldsymbol{R}^{n}, \quad t \geq 0, \quad \alpha>0 \\
& \left\{\begin{array}{l}
u(x, 0)=\phi(x) \\
u_{t}(x, 0)=\psi(x) .
\end{array}\right.
\end{aligned}
$$

We put $\alpha=1$ without loss of generality. We suppose $\phi \in H^{s+1}, \psi \in H^{s}$ and put

$$
\left\|D_{x}^{s+1} \phi\right\|+\left\|D_{x}^{s} \psi\right\|=\varepsilon
$$

By using the equation (1), we can determine

$$
\left(\frac{\partial}{\partial t}\right)^{k} u(x, 0) \quad(2 \leq k \leq s+1)
$$


successively beginning with $\phi, \psi$ and it follows

$$
\left\|D^{s+1} u(0)\right\| \leq \varepsilon h_{4}(\varepsilon)
$$

where $h_{4}$ depends only on $h_{0}$ and $h_{1}$. Then we have the following

Theorem 1 (local existence). We suppose Assumptions 1 and 3. Moreover we suppose $\phi \in H^{s+1}, \psi \in H^{s}$ and that $D^{s+1} u(0)$ satisfies

$$
\left\|D^{s+1} u(0)\right\| \leq c_{3} e
$$

where $c_{3}=\left(\frac{c_{1}}{4 c_{2}}\right)^{\frac{1}{2}}$ and $0<e \leq e_{0}$. Then there exists a positive constant $t_{0}$ such that Cauchy problem for (1) has a unique solution

$$
u(x, t) \in \mathscr{D}\left(0, t_{0} \mid e\right) \text {. }
$$

Remark. This Theorem is due to the Theorem in Chapter $\mathrm{V}$ of Dionne [1], although we modified the formulation. We only note the following: If $v \in \mathscr{D}\left(0, t_{0} \mid e\right)$, the linear equation

$$
u_{t t}-\sum a_{i j}(x, t, D v) u_{i j}+u_{t}=-b(D v)
$$

is strictly hyperbolic on $0 \leq t \leq t_{0}$ so that we get the energy inequality

$$
\left\|D^{s+1} u(t)\right\|^{2} \leq \exp (c t)\left\{\frac{c_{2}}{c_{1}}\left\|D^{s+1} u(0)\right\|^{2}+\int_{0}^{t}\left\|D^{s} b(D v)\right\|^{2} d \tau\right\}
$$

where $c$ depends only on $h_{0}$ and $e_{0}$. On the other hand, it follows from $v \in \mathscr{D}\left(0, t_{0} \mid e\right)$ that

$$
\left\|D^{s} b(D v(\tau))\right\|^{2} \leq c e^{2} \quad \text { for } \quad 0 \leq \tau \leq t_{0}
$$

Choosing $t_{0}$ sufficiently small in the above inequalities, we have

$$
\sup _{0 \leq t \leq t_{0}}\left\|D^{s+1} u(t)\right\| \leq e \quad \text { for } \quad D^{s+1} u(0) \text { as in the Theorem. }
$$

Therefore we can perform the iteration arguments. For more details, refer to the Appendix.

In order to show the global existence, we establish the following

Lemma 6 (a priori estimate). We suppose Assumptions 1 4. More- 
over we suppose that (1) has the solution $u \in \mathscr{D}(0, T \mid e)(T$ is any positive constant) for

$$
\left\{\begin{array}{l}
\left\|D_{x}^{s+1} \phi\right\|+\left\|D_{x}^{s} \psi\right\|=\varepsilon \\
\sup _{\mathbb{R}^{n \times R^{1}}} \sum\left|\bar{D}^{s+1} a_{i j}(x, t, 0)\right|=\varepsilon_{1} .
\end{array}\right.
$$

Then there exist the positive constants $\delta_{0}, \delta_{1}(e)$ such that

$$
u \in \mathscr{D}\left(0, T \mid c_{3} e\right) \quad \text { for } \quad 0<e, \varepsilon_{1} \leq \delta_{0}, 0<\varepsilon \leq \delta_{1}(e)
$$

Here $\delta_{0}, \delta_{1}$ do not depend on $T$.

Proof. For $u \in \mathscr{D}(0, T \mid e) \cap \mathscr{E}_{t}^{i}\left(H^{s+2-i}\right)(0 \leq i \leq s+2)$ and $v \in \mathscr{D}(0, T \mid e)$ we first estimate the following

$$
\begin{aligned}
I= & \int D^{s}\left\{L_{v}(u)+b(D u)\right\} \cdot D^{s} u_{t} d x=\int D^{s} u_{t t} \cdot D^{s} u_{t} d x \\
& -\int D^{s} \sum a_{i j}(D v) u_{i j} \cdot D^{s} u_{t} d x+\int D^{s} u_{t} \cdot D^{s} u_{t} d x+\int D^{s} b(D u) \cdot D^{s} u_{t} d x \\
= & I_{1}-I_{2}+I_{3}+I_{4}
\end{aligned}
$$

where

$$
L_{v}(u)=u_{t t}-\sum a_{i j}(x, t, D v) u_{i j}+u_{i}
$$

By Lemmas 4 and 5, we have

$$
\begin{aligned}
I_{1}= & \frac{1}{2} \frac{d}{d t} \int\left|D^{s} u_{t}\right|^{2} d x \\
-I_{2}= & \frac{1}{2} \frac{d}{d t} \int \sum a_{i j}(D v) D^{s} u_{i} \cdot D^{s} u_{j} d x+\int \sum\left\{D^{s}\left(a_{i j} u_{i j}\right)-a_{i j} D^{s} u_{i j}\right\} \cdot D^{s} u_{t} d x \\
& -\int \frac{1}{2} \sum\left(a_{i j}(D v) D^{s} u_{i} \cdot D^{s} u_{j}\right)_{t}+\sum a_{i j}(D v) D^{s} u_{i} \cdot D^{s} u_{j t} d x \\
& +\int \sum\left(a_{i j}(D v) D^{s} u_{i} \cdot D^{s} u_{t}\right)_{j}-\sum a_{i j}(D v) D^{s} u_{i j} \cdot D^{s} u_{t} \\
& -\sum a_{i j}(D v) D^{s} u_{i} \cdot D^{s} u_{j t} d x \\
\geq & \frac{1}{2} \frac{d}{d t} \int \sum a_{i j}(D v) D^{s} u_{i} \cdot D^{s} u_{j} d x
\end{aligned}
$$




$$
\begin{aligned}
& -c\left\{\left\|D^{s+1} v\right\| h_{0}\left(\|D v\|_{\infty}\right)+\left\|D^{s+1} v\right\|^{s} h_{0}\left(\|D v\|_{\infty}\right)+\varepsilon_{1}\right\}\left\|\bar{D}^{s+1} u\right\|^{2} \\
I_{3}= & \int\left|D^{s} u_{t}\right|^{2} d x=\left\|D^{s} u_{t}\right\|^{2} \\
I_{4}= & \int \bar{D}^{s} b(D u) \cdot \bar{D}^{s} u_{t}+b(D u) u_{t} d x \\
\geq & \int b_{1}(u) u_{t} d x-\left\|\bar{D}^{s} b(D u)\right\|\left\|\bar{D}^{s} u_{t}\right\|-\left\|b_{2}(D u)\right\|\left\|u_{t}\right\| \\
\geq & \frac{d}{d t} \int B(u) d x-c\left(1+\left\|D^{s+1} u\right\|^{s-1}\right)\left\|D^{s+1} u\right\|\left\|\bar{D}^{s+1} u\right\|^{2} h_{1}\left(\|D u\|_{\infty}\right)
\end{aligned}
$$

where $B(u)=\int_{0}^{u} b_{1}(v) d v$. From the above estimates, we have

$$
\begin{aligned}
I \geq & \frac{d}{d t}\left\{\int \frac{1}{2}\left|D^{s} u_{t}\right|^{2}+\frac{1}{2} \sum a_{i j}(D v) D^{s} u_{i} D^{s} u_{j}+B(u) d x\right\} \\
& +\left\|D^{s} u_{t}\right\|^{2}-c\left\{\varepsilon_{1}+\left(\left\|D^{s+1} v\right\|+\left\|D^{s+1} v\right\|^{s}\right) h_{0}\left(\|D v\|_{\infty}\right)\right. \\
& \left.+\left(\left\|D^{s+1} u\right\|+\left\|D^{s+1} u\right\|^{s}\right) h_{1}\left(\|D u\|_{\infty}\right)\right\}\left\|\bar{D}^{s+1} u\right\|^{2}
\end{aligned}
$$

Next we estimate

$$
\begin{aligned}
I^{\prime}= & \int D^{s}\left\{L_{v}(u)+b(D u)\right\} \cdot D^{s} u d x=\int D^{s} u_{t t} \cdot D^{s} u d x \\
& -\int D^{s}\left(\sum a_{i j} u_{i j}\right) \cdot D^{s} u d x+\int D^{s} u_{t} \cdot D^{s} u d x+\int D^{s} b(D u) \cdot D^{s} u d x \\
= & I_{1}^{\prime}-I_{2}^{\prime}+I_{3}^{\prime}+I_{4}^{\prime} .
\end{aligned}
$$

We have

$$
\begin{aligned}
I_{1}^{\prime}= & \frac{d}{d t} \int D^{s} u \cdot D^{s} u_{t} d x-\left\|D^{s} u_{t}\right\|^{2} \\
-I_{2}^{\prime}= & \int \sum a_{i j} D^{s} u_{i} \cdot D^{s} u_{j} d x \\
& +\int \sum a_{i j} D^{s} u_{i j} \cdot D^{s} u-D^{s}\left(\sum a_{i j} u_{i j}\right) \cdot D^{s} u d x \\
& +\int \sum\left(a_{i j} D^{s} u_{i} \cdot D^{s} u\right)_{j}-\sum a_{i j} D^{s} u_{i} \cdot D^{s} u_{j}-\sum a_{i j} D^{s} u_{i j} \cdot D^{s} u d x
\end{aligned}
$$




$$
\begin{aligned}
\geq & a_{1} \sum\left\|D^{s} u_{i}\right\|^{2}-c\left\{\varepsilon_{1}+\left\|D^{s+1} u\right\| h_{0}\left(\|D v\|_{\infty}\right)+\left\|D^{s+1} v\right\| h_{0}\left(\|D v\|_{\infty}\right)\right. \\
& \left.+\left\|D^{s+1} v\right\|^{s} h_{0}\left(\|D v\|_{\infty}\right)\right\}\left(\left\|\bar{D}^{s+1} u\right\|^{2}+\left\|\bar{D}^{s+1} v\right\|^{2}\right) \\
I_{3}^{\prime}= & \frac{1}{2} \frac{d}{d t} \int\left|D^{s} u\right|^{2} d x \\
I_{4}^{\prime}= & \int \bar{D}^{s} b(D u) \cdot \bar{D}^{s} u+b_{1}(u) u+b_{2}(D u) u d x \\
\geq & -\left\|\bar{D}^{s} b(D u)\right\|\left\|\bar{D}^{s} u\right\|-\left\|b_{2}(D u)\right\|\|u\| \\
\geq & -c\left(\left\|D^{s+1} u\right\|+\left\|D^{s+1} u\right\|^{s}\right)\left\|\bar{D}^{s+1} u\right\|^{2} h_{1}\left(\|D u\|_{\infty}\right) .
\end{aligned}
$$

Therefore we have

$$
\begin{aligned}
I^{\prime} \geq & \frac{d}{d t}\left(\int \frac{1}{2}\left|D^{s} u\right|^{2}+D^{s} u \cdot D^{s} u_{t} d x\right)+a_{1} \sum\left\|D^{s} u_{i}\right\|^{2}-\left\|D^{s} u_{t}\right\|^{2} \\
& -c\left[\varepsilon_{1}+\left\{h_{1}\left(\|D u\|_{\infty}\right)+h_{0}\left(\|D v\|_{\infty}\right)\right\}\left(\left\|D^{s+1} u\right\|+\left\|D^{s+1} v\right\|\right.\right. \\
& \left.\left.+\left\|D^{s+1} u\right\|^{s}+\left\|D^{s+1} v\right\|^{s}\right)\right]\left(\left\|\bar{D}^{s+1} u\right\|^{2}+\left\|\bar{D}^{s+1} v\right\|^{2}\right)
\end{aligned}
$$

Choosing some positive number $0<\lambda<\frac{1}{2}$, we get from (10) and (11)

$$
\begin{aligned}
& \int_{0}^{t} I+\lambda I^{\prime} d \tau \geq\left.\left[E_{v}\{u(\tau)\}+\int B(u(\tau)) d x\right]\right|_{0} ^{t} \\
& \quad+\int_{0}^{t} \frac{1}{2}\left\|D^{s} u_{t}(\tau)\right\|^{2}+\lambda a_{1} \sum\left\|D^{s} u_{i}(\tau)\right\|^{2} \\
& \quad-c(1+\lambda)\left\{\varepsilon_{1}+\left(e+e^{s}\right) h\left(e_{0}\right)\right\}\left\{\left\|\bar{D}^{s+1} u(\tau)\right\|^{2}+\left\|\bar{D}^{s+1} v(\tau)\right\|^{2}\right\} d \tau
\end{aligned}
$$

where $h\left(e_{0}\right)=h_{0}\left(c e_{0}\right)+h_{1}\left(c e_{0}\right)$.

Now denote by $u_{\delta}(x, t)$ the function $\left(\phi_{\delta} * u\right)(x, t)$ where $\phi_{\delta} *$ is Friedrichs' mollifier with respect to $x$. Then we note that for the solution $u \in \mathscr{D}(0, T \mid e)$ of $(1)$, it follows

$$
u_{\delta}(x, t) \in \mathscr{D}(0, T \mid e) \cap \mathscr{E}_{t}^{i}\left(H^{s+2-i}\right) \quad(0 \leq i \leq s+2) .
$$

Applying $\phi_{\delta} *$ to (1),

$$
L_{u}\left(u_{\delta}\right)+b\left(D u_{\delta}\right)=C_{\delta} u+C_{\delta}^{\prime} u
$$


where

$$
\begin{aligned}
& C_{\delta} u=\Sigma\left[\phi_{\delta} *\left\{a_{i j}(D u) u_{i j}\right\}-a_{i j}(D u) u_{\delta i j}\right] \\
& C_{\delta}^{\prime} u=b\left(D u_{\delta}\right)-\phi_{\delta} * b(D u)
\end{aligned}
$$

From (12), it follows

$$
\begin{gathered}
{\left.\left[E_{u}\left\{u_{\delta}(\tau)\right\}+\int B\left(u_{\delta}(\tau)\right) d x\right]\right|_{0} ^{t}+\int_{0}^{t} \frac{1}{2}\left\|D^{s} u_{\delta t}\right\|^{2}+\lambda a_{1} \sum\left\|D^{s} u_{\delta i}\right\|^{2}} \\
-c(1+\lambda)\left\{\varepsilon_{1}+\left(e+e^{s}\right) h\left(e_{0}\right)\right\}\left(\left\|\bar{D}^{s+1} u\right\|^{2}+\left\|\bar{D}^{s+1} u_{\delta}\right\|^{2}\right) d \tau \\
\leq \int_{0}^{t}\left(\left\|D^{s} C_{\delta}^{\prime} u\right\|+\left\|D^{s} C_{\delta}^{\prime} u\right\|\right)\left(\left\|D^{s} u_{\delta t}\right\|+\lambda\left\|D^{s} u_{\delta}\right\|\right) d \tau .
\end{gathered}
$$

Then we have

$$
\begin{aligned}
& E_{u}\left\{u_{\delta}(\tau)\right\} \longrightarrow E_{u}\{u(\tau)\}, \quad \int B\left(u_{\delta}(\tau)\right) d x \longrightarrow \int B(u(\tau)) d x \\
& \left\|D^{s+1} u_{\delta}(\tau)\right\| \longrightarrow\left\|D^{s+1} u(\tau)\right\| \\
& \left\|D^{s} C_{\delta}^{\prime} u(\tau)\right\| \longrightarrow 0 \\
& \left\|D^{s} C_{\delta} u(\tau)\right\| \longrightarrow 0
\end{aligned}
$$

for every $0 \leq \tau \leq t$ when $\delta \rightarrow 0$. In fact, (13) (15) are easily verified and for (16) refer to Chapter 6 of [3]. Therefore, taking $\delta \rightarrow 0$, we get

$$
\begin{aligned}
& {\left.\left[E_{u}\{u(\tau)\}+\int B(u(\tau)) d x\right]\right|_{0} ^{t}} \\
& +\int_{0}^{t} \frac{1}{2}\left\|D^{s} u_{t}(\tau)\right\|^{2}+\lambda a_{1} \Sigma\left\|D^{s} u_{i}(\tau)\right\|^{2} \\
& -c(1+\lambda)\left\{\varepsilon_{1}+\left(e+e^{s}\right) h\left(e_{0}\right)\right\}\left\|\bar{D}^{s+1} u(\tau)\right\|^{2} d \tau \leq 0 .
\end{aligned}
$$

Choosing a small constant $\delta_{0}$ which satisfies

$$
c(1+\lambda)\left\{\varepsilon_{1}+\left(e+e^{s}\right) h\left(e_{0}\right)\right\} \leq \frac{1}{2} \min \left(\frac{1}{2}, \lambda a_{1}\right) \quad \text { for } \quad 0<\varepsilon_{1}, e \leq \delta_{0},
$$

we have 
(18) $\left.\left[E_{u}\{u(\tau)\}+\int B(u(\tau)) d x\right]\right|_{0} ^{t}+\frac{1}{2} \min \left(\frac{1}{2}, \lambda a_{1}\right) \int_{0}^{t}\left\|\bar{D}^{s+1} u(\tau)\right\|^{2} d \tau \leq 0$

Since

$$
\int B(\phi) d x \leq \int|\phi| \sup _{|v| \leq|\phi|}\left|b_{1}(v)\right| d x \leq c \varepsilon^{3} h_{1}(\varepsilon)
$$

it follows from (18) and (9) that

$$
\begin{aligned}
\left\|D^{s+1} u(t)\right\|^{2} & \leq c_{1}^{-1} E_{u}\{u(t)\} \\
& \leq c_{1}^{-1} E_{u}\{u(0)\}+c_{1}^{-1} \int B(\phi) d x \\
& \leq c_{1}^{-1} c_{2} h_{4}(\varepsilon) \varepsilon+c h_{1}(\varepsilon) \varepsilon^{3}
\end{aligned}
$$

Therefore, choosing a constant $\delta_{1}$ so small that

$$
\text { right hand side of }(19) \leq c_{3}^{2} e^{2} \quad \text { for } 0<\varepsilon \leq \delta_{1} \text {, }
$$

it follows consequently

$$
u \in \mathscr{D}\left(0, T \mid c_{3} e\right) \quad \text { for } \quad 0<e, \varepsilon_{1} \leq \delta_{0}, 0<\varepsilon \leq \delta_{1}(e) .
$$

Q.E.D.

By Theorem 1 and Lemma 6, we have the following

Theorem 2. We suppose Assumptions 1 4, that is,
i) $a_{i j}(x, t, y) \in C^{s+1}\left(\boldsymbol{R}^{n} \times \boldsymbol{R}^{1} \times \boldsymbol{R}^{n+2}\right)$.
ii) $a_{i j}=a_{j i}$.
iii) $\sum a_{i j}(x, t, y) \xi_{i} \xi_{j} \geq a(y) \sum \xi_{i}^{2}, \quad a(0)>0$
for all $x \in \boldsymbol{R}^{n}, t \in \boldsymbol{R}^{1}, y \in \boldsymbol{R}^{n+2}, \xi \in \boldsymbol{R}^{n}$
where $a(y) \in C^{0}\left(\boldsymbol{R}^{n+2}\right)$.
iv) $\left\{\begin{array}{l}\sup _{\boldsymbol{R}^{n \times R^{1}}} \sum\left|D_{x, t, y}^{s+1} a_{i j}(x, t, y)\right| \leq h_{0}(|y|) \\ \sup _{\boldsymbol{R}^{n \times R^{1}}} \sum\left|\bar{D}_{x} a_{i j}(x, t, y)\right| \leq|\tilde{y}| h_{0}(|y|) \quad \text { for } \quad|\tilde{y}| \leq 1\end{array}\right.$

v) $b(y) \in C^{s+1}\left(R^{n+2}\right), \quad\left|D_{y}^{s+1} b(y)\right| \leq h_{1}(|y|)$. 
vi) $D_{y} b(0)=0$.

vii) $b(D u)=b_{1}(u)+b_{2}(D u)$,

$$
\left\{\begin{array}{l}
b_{1}(u) u \geq 0 \\
\left|b_{2}(y)\right| \leq|\tilde{y}|^{2} h_{1}(|y|) \quad \text { for } \quad|\tilde{y}| \leq 1 .
\end{array}\right.
$$

Here $s$ represents $\left[\frac{n}{2}\right]+2$. Moreover we suppose $\phi \in H^{s+1}, \psi \in H^{s}$ and put

$$
\left\{\begin{array}{l}
\left\|D_{x}^{s+1} \phi\right\|+\left\|D_{x}^{s} \psi\right\|=\varepsilon \\
\sup _{\boldsymbol{R}^{n \times R^{1}}} \sum\left|\bar{D}^{s} a_{i j}(x, t, 0)\right|=\varepsilon_{1} .
\end{array}\right.
$$

Then there exists a positive constant $\varepsilon_{0}$ such that the Cauchy problem for

$$
\begin{aligned}
& u_{t t}-\sum a_{i j}(x, t, D u) u_{i j}+u_{t}+b(D u)=0 \\
& \left\{\begin{array}{l}
u(0)=\phi \\
u_{t}(0)=\psi
\end{array}\right.
\end{aligned}
$$

has a unique solution $u(x, t) \in \mathscr{D}\left(0,+\infty \mid e_{0}\right)$ for $0<{ }^{\forall} \varepsilon,{ }^{\forall} \varepsilon_{1} \leq \varepsilon_{0}$. Furthermore u satisfies

$$
\|u(t)\|_{\infty}+\left\|\bar{D}^{s} u(t)\right\| \longrightarrow 0 \text { as } t \longrightarrow+\infty
$$

Corollary 1. In addition to the assumptions of Theorem 2, we further suppose the following:
i) $B(u)=\int_{0}^{u} b_{1}(v) d v \leq u b_{1}(u) h_{5}(|u|)$.
ii) $\left|b_{2}(y)\right| \leq\left|y_{2}\right||\tilde{y}| h_{1}(|y|)$.
iii) $\sum a_{i j} u_{i j}=\sum a_{i j}^{\prime}(D u) u_{i j}+\sum\left\{f^{i}\left(u_{i}\right)\right\}_{i}$

$$
\left\{\begin{array}{l}
f^{i}(0)=0, \quad \frac{\partial}{\partial u_{i}} f^{i}(0)=0 \\
\sum_{k \neq 2}\left|\frac{\partial}{\partial y_{k}} a_{i j}^{\prime}(y)\right| \leq\left|y_{2}\right| h_{0}(|y|) .
\end{array}\right.
$$


Then solution $u$ that is obtained by the Theorem 2 satisfies

$$
\|\bar{D} u(t)\|^{2}+\int B(u(t)) d x \leq c t^{-1}
$$

Corollary 2. In addition to the assumptions of Theorem 2, we further suppose the following:

i) $b_{1}(u)=0$.

ii) $\left\{\begin{array}{l}\left|D_{y_{1}}^{s} b_{2}(y)\right| \leq\left|y_{2}\right|^{2} h_{1}(|y|) \\ \left|\frac{\partial}{\partial \tilde{y}} b_{2}(y)\right| \leq\left|y_{2}\right| h_{1}(|y|) .\end{array}\right.$

iii) $\sum a_{i j} u_{i j}=\sum a_{i j}^{\prime}(D u) u_{i j}+\sum\left\{f^{i}\left(u_{i}\right)\right\}_{i}$

$$
\left\{\begin{array}{l}
f^{i}(0)=0, \quad \frac{\partial}{\partial u_{i}} f^{i}(0)=0 \\
\sum_{k \neq 2}\left|\frac{\partial}{\partial y_{k}} a_{i j}^{\prime}(y)\right| \leq\left|y_{2}\right| h_{0}(|y|) .
\end{array}\right.
$$

Then the solution $u$ that is obtained by Theorem 2 satisfies

$$
\begin{aligned}
\left\|\bar{D}^{s+1} u(t)\right\|^{2} & \leq c t^{-1}, \\
\|u(t)\|_{\infty} & \leq c t^{-\gamma} \quad\left(\gamma=\frac{n}{4(s-1)}\right) .
\end{aligned}
$$

Proof of the Theorem 2. From (9) and Theorem 1, we can choose a positive constant $\delta_{2}$ as

$$
u \in \mathscr{D}\left(0, t_{0} \mid e\right), \quad 0<e \leq \delta_{0} \quad \text { for } \quad 0<{ }^{\forall} \varepsilon \leq \delta_{2} .
$$

Now if we choose $\varepsilon_{0}=\min \left(\delta_{2}, \delta_{1}(e)\right)$, it follows by Lemma 6

$$
u \in \mathscr{D}\left(0, t_{0} \mid c_{3} e\right) \quad \text { for } 0<{ }^{\forall} \varepsilon,{ }^{\forall} \varepsilon_{1} \leq \varepsilon_{0} .
$$

By using Theorem 1 again with $\phi=u\left(t_{0}\right), \psi=u_{t}\left(t_{0}\right)$, the solution

$$
u \in \mathscr{D}\left(0,2 t_{0} \mid e\right)
$$

exists for $0<{ }^{\forall} \varepsilon,{ }^{\forall} \varepsilon_{1} \leq \varepsilon_{0}$. By Lemma 6, (20) immediately implies 


$$
u \in \mathscr{D}\left(0,2 t_{0} \mid c_{3} e\right)
$$

Thus repeating the same arguments, we have the solution

$$
u \in \mathscr{D}\left(0,+\infty \mid c_{3} e\right) \subset \mathscr{D}\left(0,+\infty \mid e_{0}\right) .
$$

Next, by the same way as we got (18), we have

$$
\left\|\bar{D}^{s+1} u(t)\right\|^{2}+\int_{0}^{t}\left\|\bar{D}^{s+1} u(\tau)\right\|^{2}+\int b_{1}(u(\tau)) u(\tau) d x d \tau \leq c .
$$

(21) and the following Nirenberg's inequality ([5])

$$
\|u\|_{\infty} \leq c\left\|\bar{D}_{x}^{s-1} u\right\|^{\alpha}\|u\|^{1-\alpha}, \quad \alpha=\frac{n}{2(s-1)}
$$

give

$$
\left\|\bar{D}^{s} u(t)\right\|+\|u(t)\|_{\infty} \longrightarrow 0 \text { as } t \longrightarrow+\infty
$$

Proof of the Corollary 1. Define $E_{1}(t)$ by

$$
E_{1}(t)=\int \frac{1}{2} u_{t}^{2}+\frac{1}{2} \sum a_{i j}^{\prime}(0) u_{i} u_{j}+\sum F^{i}\left(u_{i}\right)+B(u) d x
$$

where

$$
F^{i}\left(u_{i}\right)=\int_{0}^{u_{i}} f^{i}(v) d v
$$

Estimating

$$
\int L(u) u_{t} d x=0
$$

by using the assumptions, it follows that

$$
\frac{d}{d t} E_{1}(t)+\gamma\left\|u_{t}(t)\right\|^{2} \leq 0 \quad(\gamma>0) \quad \text { for } \quad 0<{ }^{\forall} \varepsilon \leq \varepsilon_{0}
$$

which implies

$$
E_{1}(t) \leq E_{1}(\tau) \quad \text { for } \quad t \geq \tau
$$


It follows by integrating (23) and using (21) that

$$
\begin{aligned}
t E_{1}(t) & \leq \int_{0}^{t} E_{1}(\tau) d \tau \\
& \leq \int_{0}^{t} c\|\bar{D} u(\tau)\|^{2}+\int c b_{1}(u(\tau)) u(\tau) d x d \tau \\
& \leq c
\end{aligned}
$$

which gives

$$
\|\bar{D} u(t)\|^{2}+\int B(u(t)) d x \leq c t^{-1}
$$

Q.E.D.

Proof of the Corollary 2. For this case we can give a proof by estimating

$$
\int D^{s}\{L(u)\} \cdot D^{s} u_{t}+\lambda \bar{D}^{s}\{L(u)\} \cdot \bar{D}^{s} u d x=0 \quad(0<\lambda<1)
$$

similarly as in the previous arguments and using Nirenberg's inequality (22). We omit the details.

Q.E.D.

\section{§3. Initial-Boundary Value Problem and Periodic Solutions}

We consider the following initial-boundary value problem

$(1)^{\prime}$

$$
\begin{aligned}
& L(u)=\varepsilon_{2} f(x, t) \quad x \in \Omega, t \geq 0,0<\varepsilon_{2} \leq 1 \\
& \left\{\begin{array}{l}
u(0)=\phi \\
u_{t}(0)=\psi \\
\left.u\right|_{\partial \Omega}=0
\end{array}\right.
\end{aligned}
$$

where $\Omega$ is a bounded open set in $\boldsymbol{R}^{n}$ with $C^{\infty}$-boundary $\partial \Omega$. For the term $f(x, t)$, we assume

\section{Assumption 5.}

i) $f(x, t) \in C^{s}\left(\boldsymbol{R}^{n} \times \boldsymbol{R}^{1}\right)$. 
ii) $\sup _{t \in \mathbb{R}^{1}}\left\|D^{s} f(t)\right\| \leq M<+\infty$.

Moreover we assume the following compatibility condition;

$$
u^{k-1} \in H^{s-k+2} \cap \stackrel{\circ}{H}^{s-k+1}, u^{k} \in \stackrel{H}{ }^{s-k+1} \quad \text { for } \quad 1 \leq k \leq s
$$

where

$$
u^{k}=\left(\frac{d}{d t}\right)^{k} u(x, 0)
$$

which are determined successively by (1)' beginning with $\phi$ and $\psi$. We note that if $u \in \mathscr{D}\left(0, T \mid e_{0}\right)$ is the solution of $(1)^{\prime}$, it follows

(25) $\left\|D^{k+1} u\right\| \leq c\left(h_{0}, h_{1}, e_{0}, a_{1}, c_{0}, M, \Omega\right)\left(\left\|D_{t}^{k+1} u\right\|+\left\|D^{1, k} u\right\|+\varepsilon_{2}\left\|D^{s} f\right\|\right)$

$$
\text { for } 1 \leq k \leq s
$$

by using the Lemmas 4 and 5 (especially v) of Lemma 4). We show the local arguments to the simple case $(b=0, f=0$ and $\alpha=0)$ at the Appendix.

We have the following

Theorem 3. We suppose Assumptions 1, 3 and 5, that is,

i) $a_{i j}(x, t, y) \in C^{s+1}\left(\boldsymbol{R}^{n} \times \boldsymbol{R}^{1} \times \boldsymbol{R}^{n+2}\right)$.

ii) $a_{i j}=a_{j i}$.

iii) $\sum a_{i j}(x, t, y) \xi_{i} \xi_{j} \geq a(y) \sum \xi_{i}^{2}, \quad a(0)>0$

for all $x \in \mathbb{R}^{n}, t \in \mathbb{R}^{1}, y \in \mathbb{R}^{n+2}, \xi \in \mathbb{R}^{n}$

where $a(y) \in C^{0}\left(\boldsymbol{R}^{n+2}\right)$.

iv) $\sup _{\mathbb{R}^{n} \times R^{1}} \sum\left|D_{x, t, y}^{s+1} a_{i j}(x, t, y)\right| \leq h_{0}(|y|)$.

v) $b(y) \in C^{s+1}\left(\boldsymbol{R}^{n+2}\right), \quad\left|D_{y}^{s+1} b(y)\right| \leq h_{1}(|y|)$.

vi) $D_{y} b(0)=0$.

vii) $\left\{\begin{array}{l}f(x, t) \in C^{s}\left(\boldsymbol{R}^{n} \times \boldsymbol{R}^{1}\right) \\ \sup _{t \in \mathbb{R}^{1}}\left\|D^{s} f(t)\right\| \leq M<+\infty .\end{array}\right.$ 
Here $s$ represents $\left[\frac{n}{2}\right]+2$. Moreover we suppose $\phi \in H^{s+1} \cap H^{s}$ and $\psi \in \stackrel{\circ}{H}^{s}$ satisfy the compatibility condition (24), and put

$$
\left\{\begin{array}{l}
\left\|D_{x}^{s+1} \phi\right\|+\left\|D_{x}^{s} \psi\right\|=\varepsilon \\
\sup _{\mathbb{R}^{n} \times \boldsymbol{R}^{1}} \sum\left|\bar{D}^{s} a_{i j}(x, t, 0)\right|=\varepsilon_{1} .
\end{array}\right.
$$

Then there exists a positive constant $\varepsilon_{0}$ such that the initial-boundary value problem for

$$
\begin{aligned}
& u_{t t}-\sum a_{i j}(x, t, D u) u_{i j}+u_{t}+b(D u)=\varepsilon_{2} f(x, t) \\
& \left\{\begin{array}{l}
u(0)=\phi \\
u_{t}(0)=\psi \\
\left.u\right|_{\partial \Omega}=0
\end{array}\right.
\end{aligned}
$$

has a unique solution $u(x, t)$ which satisfies

$u(x, t) \in \mathscr{D}\left(0,+\infty \mid e_{0}\right) \quad$ and $D_{t}^{s} u(x, t) \in \mathscr{E}_{i}^{\circ}\left(\stackrel{H}{ }^{1}\right) \quad$ for $0<{ }^{\forall} \varepsilon,{ }^{\forall} \varepsilon_{1},{ }^{\forall} \varepsilon_{2} \leq \varepsilon_{0}$.

Furthermore $u$ satisfies

$$
\left\|D^{s+1} u(t)\right\| \leq c\left\|D^{s+1} u(0)\right\| \exp (-\gamma t)+c \varepsilon_{2} \sup _{t \in \mathbb{R}^{1}}\left\|D^{s} f(t)\right\|
$$

where $\gamma$ is some positive constant.

Corollary 3 (Periodic solutions). We suppose Assumptions 1, 3 and 5. Moreover we suppose that $a_{i j}(x, t, y)$ and $f(x, t)$ are $\omega$-time-periodic, that is,

$$
a_{i j}(x, t+\omega, y)=a_{i j}(x, t, y), \quad f(x, t+\omega)=f(x, t)
$$

for all $x, t, y$. Then there exists a positive constant $\varepsilon_{0}$ such that

$$
\begin{aligned}
& L(u)=\varepsilon_{2} f(x, t) \quad x \in \Omega, t \in \mathbb{R}^{1} \\
& \left.u\right|_{\partial \Omega}=0
\end{aligned}
$$

has a unique $\omega$-time-periodic solution $u(x, t)$ which satisfies

$$
\begin{aligned}
& u(x, t) \in \mathscr{D}\left(-\infty,+\infty \mid e_{0}\right) \text { and } D_{t}^{s} u(x, t) \in \mathscr{E}_{t}^{0}\left(\stackrel{\circ}{H}^{1}\right) \quad \text { for } \\
& 0<{ }^{\forall} \varepsilon_{1},{ }^{\forall} \varepsilon_{2} \leq \varepsilon_{0} .
\end{aligned}
$$


Furthermore, for the time periodic solution $u(x, t)$ as we got above, any solution $v(x, t)$ of $(1)^{\prime \prime}$ which satisfies

$$
\left\{\begin{array}{l}
v(x, t) \in \mathscr{D}\left(0,+\infty \mid e_{0}\right) \text { and } D_{t}^{s} v(x, t) \in \mathscr{E}_{t}^{0}\left(\stackrel{H}{ }^{1}\right) \\
\left\|D^{s+1} v(0)^{s}\right\|=\varepsilon
\end{array}\right.
$$

is asymptotic to $u(x, t)$ exponentially as $t \rightarrow+\infty$, that is,

$$
\left\|D^{s}(u-v)(t)\right\| \leq c \exp (-\gamma t) \quad(\gamma>0)
$$

for $0<{ }^{\forall} \varepsilon,{ }^{\forall} \varepsilon_{1},{ }^{\forall} \varepsilon_{2} \leq \varepsilon_{0}$.

Proof of Theorem 3. Recalling the arguments in $\S 2$, it is sufficient only to show the apriori estimate for

$$
u \in \mathscr{D}(0,+\infty \mid e) \cap \mathscr{E}_{t}^{\infty}\left(H^{s+1}\right) \cap \mathscr{E}_{t}^{\infty}\left(\stackrel{\circ}{ }^{s}\right) .
$$

If we want to show the estimate for $u \in \mathscr{D}(0,+\infty \mid e)$ and $D_{t}^{s} u \in \mathscr{E}_{t}^{0}\left(\dot{H}^{1}\right)$, we may use the mollifier with respect to $t$ for this case. Then estimating

$$
\begin{gathered}
\int D_{t}^{s}\{L(u)\} \cdot D_{t}^{s} u_{t} d x+\lambda \int D_{t}^{s}\{L(u)\} \cdot D_{t}^{s} u d x \\
=\varepsilon_{2} \int D_{t}^{s} f \cdot D_{t}^{s} u_{t} d x+\lambda \varepsilon_{2} \int D_{t}^{s} f \cdot D_{t}^{s} u d x
\end{gathered}
$$

by the same way as in Theorem 2 , we get

$$
\begin{aligned}
& \frac{d}{d t}\{E(t)\}+\frac{1}{2}\left\|\bar{D}_{t}^{s+1} u(t)\right\|^{2}+\lambda a_{1}\left\|\bar{D}^{1, s} u(t)\right\|^{2} \\
& -c(1+\lambda)\left\{\varepsilon_{1}+\varepsilon_{2}+\left(e+e^{s}\right) h\left(e_{0}\right)\right\}\left\|D^{s+1} u(t)\right\|^{2} \leq c \varepsilon_{2}(1+\lambda)\left\|D_{t}^{s} f(t)\right\|^{2}
\end{aligned}
$$

where

$$
\begin{aligned}
E(t)= & \int \frac{\lambda}{2}\left|D_{t}^{s} u\right|^{2}+\lambda D_{t}^{s} u \cdot D_{t}^{s} u_{t}+\frac{1}{2}\left|D_{t}^{s} u_{t}\right|^{2} \\
& +\frac{1}{2} \sum a_{i j}(x, t, D u) D_{t}^{s} u_{i} \cdot D_{t}^{s} u_{j} d x \quad\left(0<\lambda<\frac{1}{2}\right) .
\end{aligned}
$$

By Poincaré's inequality 


$$
\|u\| \leq c(\Omega)\left\|\bar{D}_{x} u\right\|
$$

and (25), it follows

$$
\left\|D^{s+1} u\right\| \leq c\left(\left\|\bar{D}_{t}^{s+1} u\right\|+\left\|\bar{D}^{1, s} u\right\|+\varepsilon_{2}\left\|D^{s} f\right\|\right) .
$$

Therefore choosing $\delta$ small, (26) implies

$$
\begin{aligned}
& \frac{d}{d t}\{E(t)\}+\frac{1}{4}\left\|\bar{D}_{t}^{s+1} u(t)\right\|^{2}+\frac{\lambda}{2} a_{1}\left\|\bar{D}^{1, s} u(t)\right\|^{2} \\
& \leq c \varepsilon_{2}\left\|D^{s} f(t)\right\|^{2} \quad \text { for } \quad 0<e, \varepsilon_{1}, \varepsilon_{2} \leq \delta .
\end{aligned}
$$

By Lemma 2 and (27), we get

$$
E(t) \leq c\left\{\left\|\bar{D}_{t}^{s+1} u(t)\right\|^{2}+\left\|\bar{D}^{1, s} u(t)\right\|^{2}\right\}
$$

so that by (28)

$$
\frac{d}{d t}\{E(t)\}+2 \gamma E(t) \leq c \varepsilon_{2}\left\|D^{s} f(t)\right\|^{2} \quad(\gamma>0)
$$

which implies

$$
E(t) \leq c E(0) \exp (-2 \gamma t)+c \varepsilon_{2} \sup _{t \in R^{1}}\left\|D^{s} f(t)\right\|^{2} .
$$

Hence we get the estimate

$$
\left\|D^{s+1} u(t)\right\| \leq c\left\|D^{s+1} u(0)\right\| \exp (-\gamma t)+c \varepsilon_{2} \sup _{t \in R^{1}}\left\|D^{s} f(t)\right\|
$$

which become apriori estimate.

Q.E.D.

Proof of Corollary 3. We consider the following initial-boundary value problem;

$$
\begin{aligned}
& L\left(u^{m}\right)=\varepsilon_{2} f^{m}(x, t) \quad(m=0,1,2, \ldots) \\
& \left\{\begin{array}{l}
u^{m}(x,-m)=0 \\
u_{t}^{m}(x,-m)=0 \\
\left.u^{m}\right|_{\partial \Omega}=0
\end{array}\right.
\end{aligned}
$$

where $f^{m}(x, t)$ satisfies the following conditions: 
i) $f^{m}(x, t) \in C^{s}\left(\boldsymbol{R}^{n} \times \boldsymbol{R}^{1}\right)$.

ii) $\sup _{t \in \mathbb{R}^{1}}\left\|D^{s} f^{m}(t)\right\| \leq c M$ where $c$ is independent of $m$.

ii) $\begin{cases}f^{m}(x, t) \equiv f(x, t) & \text { for } t \geq-m+1, \\ D^{s} f^{m}(x, t) \equiv 0 & \text { for } t \leq-m\end{cases}$

Applying Theorem 3 to (29), we have the solution of (29) as

$$
\left\{\begin{array}{l}
u^{m}(x, t) \in \mathscr{D}\left(-m,+\infty \mid e_{0}\right) \quad \text { and } \quad D_{t}^{s} u^{m}(x, t) \in \mathscr{E}_{t}^{0}\left(\dot{H}^{1}\right) \\
\sup _{t \geq-m}\left\|D^{s+1} u^{m}(t)\right\| \leq c \varepsilon_{2} \quad \text { for } 0<\varepsilon_{1}, \varepsilon_{2} \leq{ }^{\exists} \varepsilon_{0}
\end{array}\right.
$$

where we emphasize that $c$ and $\varepsilon_{0}$ are independent of $m$. Putting $u^{m}(x$, $t) \equiv 0$ for $t \leq-m$ we can extend $u^{m}(x, t)$ on $-\infty<t<+\infty$ as

$$
\left\{\begin{array}{l}
u^{m}(x, t) \in \mathscr{D}\left(-\infty,+\infty \mid e_{0}\right) \text { and } D_{t}^{s} u^{m}(x, t) \in \mathscr{E}_{t}^{0}\left(\stackrel{H}{ }^{1}\right) \\
\sup _{t \in \boldsymbol{R}^{1}}\left\|D^{s+1} u^{m}(t)\right\| \leq c \varepsilon_{2} \quad \text { for } 0<\varepsilon_{1}, \varepsilon_{2} \leq \varepsilon_{0} .
\end{array}\right.
$$

Then estimating

$$
\begin{array}{r}
\int D_{t}^{s-1}\left\{L\left(u^{m+1}\right)-L\left(u^{m}\right)\right\} \cdot\left\{D_{t}^{s-1}\left(u_{t}^{m+1}-u_{t}^{m}\right)+\lambda D_{t}^{s-1}\left(u^{m+1}-u^{m}\right)\right\} d x=0 \\
(t \geq-m+1)
\end{array}
$$

by using Lemmas 4,5 and (27) as before, we have

$$
\frac{d}{d t}\left\{E_{u^{m}}\left(u^{m+1}-u^{m}\right)\right\}+2 \gamma E_{u^{m}}\left(u^{m+1}-u^{m}\right) \leq 0
$$

for $0<\varepsilon_{1}, \varepsilon_{2} \leq{ }^{\exists} \varepsilon_{0}$ and $t \geq-m+1$ where $\gamma$ is some positive constant independent of $m$ and

$$
\begin{aligned}
E_{v}(u)=\int \frac{\lambda}{2}\left|D_{t}^{s-1} u\right|^{2} & +\lambda D_{t}^{s-1} u \cdot D_{t}^{s-1} u_{t}+\frac{1}{2}\left|D_{t}^{s-1} u_{t}\right|^{2} \\
& +\frac{1}{2} \sum a_{i j}(x, t, D v) D_{t}^{s-1} u_{i} \cdot D_{t}^{s-1} u_{j} d x
\end{aligned}
$$

From (32), we have

$$
\left\|D^{s}\left(u^{m+1}-u^{m}\right)(t)\right\| \leq c\left\|D^{s}\left(u^{m+1}-u^{m}\right)(\tau)\right\| \exp \{-\gamma(t-\tau)\}
$$

for all $t \geq \tau \geq-m+1$. Let $T$ be any fixed finite number. Then we can 
suppose $T \geq-m+1$ by taking $m$ large. So it follows from (31) and (33)

$$
\left\|D^{\mathrm{s}}\left(u^{m+1}-u^{m}\right)(T)\right\| \leq c \exp \{-\gamma(T+m-1)\} .
$$

This gives

$$
\left\|D^{s}\left(u^{m+1}-u^{m}\right)(T)\right\| \longrightarrow 0 \text { as } m \longrightarrow+\infty .
$$

Moreover we have from (33)

$$
\left\|D^{s}\left(u^{m+1}-u^{m}\right)(t)\right\| \leq c\left\|D^{s}\left(u^{m+1}-u^{m}\right)(T)\right\| \exp \{-\gamma(t-T)\} \quad \text { for } \quad t \geq T \text {. }
$$

Therefore it follows from (34) and (35) that

$$
\sup _{t \geq T}\left\|D^{s}\left(u^{m+1}-u^{m}\right)(t)\right\| \longrightarrow 0 \quad \text { as } \quad m \longrightarrow+\infty
$$

for any fixed finite number $T$. On the other hand, it is clear that

$$
\sup _{t \geq T}\left\|D^{s}\left(f^{m}-f\right)(t)\right\| \longrightarrow 0 \quad m \longrightarrow+\infty
$$

for any finite number $T$. Hence (31), (36) and (37) give the existence of a solution of (1)" (refer to the last of Appendix for the regularity).

Now we will show the uniqueness. We suppose two solutions $u$ and $v$ to $(1)^{\prime \prime}$ exist. Then putting $w=u-v$, we have

$$
\left\|D^{s} w(t)\right\| \leq c\left\|D^{s} w(\tau)\right\| \exp \{-\gamma(t-\tau)\} \quad \text { for } \quad t \geq \tau
$$

by the same way as we got (33). Now if $w \not \equiv 0$, there exists some $t_{0}$ such that

$$
\left\|D^{s} w\left(t_{0}\right)\right\| \neq 0
$$

From (38) we get

$$
\left\|D^{s_{w}}\left(t_{0}\right)\right\| \leq c \exp \left\{-\gamma\left(t_{0}-\tau\right)\right\} \quad \text { for all } \tau \leq t_{0} .
$$

If we choose $\tau$ negatively large enough, (40) contradicts to (39) and this implies the uniqueness. Therefore from the existence and uniqueness, it is clear that if $a_{i j}$ and $f$ are periodic, the solution is periodic. Finally we can get the stability from (38).

Q.E.D. 


\section{Appendix}

We consider the local solution of the following initial-boundary value problem;

$$
\begin{aligned}
& u_{t t}-\sum_{i, j=1}^{n} a_{i j}(x, t, D u) u_{i j}=0 \quad x \in \Omega \quad t \geq 0 \\
& \left\{\begin{array}{l}
u(x, 0)=\phi \\
u_{t}(x, 0)=\psi \\
\left.u\right|_{\partial \Omega}=0
\end{array}\right.
\end{aligned}
$$

where $\Omega$ is $\boldsymbol{R}^{n}$ or a bounded open set in $\boldsymbol{R}^{n}$ with $C^{\infty}$-boundary $\partial \Omega$. We assume $\phi$ and $\psi$ satisfies the compatibility condition in the sense of (24).

First we consider the following linear problem;

$$
\begin{aligned}
& L_{v}(u) \equiv u_{t t}-\sum_{i, j} a_{i j}(x, t, D v(x, t)) u_{i j}=f(x, t) \quad x \in \Omega \quad t \geq 0 \\
& \left\{\begin{array}{l}
u(x, 0)=\phi \\
u_{t}(x, 0)=\psi \\
\left.u\right|_{\partial \Omega}=0 .
\end{array}\right.
\end{aligned}
$$

Then we have the following

Proposition 1. We suppose Assumption 1. Moreover we suppose that $\phi \in H^{s+1} \cap \stackrel{\circ}{H}^{s}$ and $\psi \in \stackrel{\circ}{H}^{s}$ satisfy the compatibility condition and that

$$
\left\{\begin{array}{l}
v \in \mathscr{D}(0,+\infty \mid e) \\
f \in \mathscr{E}_{t}^{i}\left(H^{s-i}\right) \quad(0 \leq i \leq s) .
\end{array}\right.
$$

Then (42) has a unique solution $u(x, t)$ which satisfies

$$
u(x, t) \in \mathscr{E}_{t}^{0}\left(H^{s+1} \cap \stackrel{\circ}{ }^{s}\right) \cap \mathscr{E}_{t}^{i}\left(\stackrel{\circ}{ }^{s+1-i}\right) \quad(1 \leq i \leq s+1)
$$

and the following inequality holds: For $1 \leq l \leq s$ 
(44) $\left\|D^{l+1} u(t)\right\|^{2} \leq c \exp (c t)\left\{\left\|D^{l+1} u(0)\right\|^{2}+\left\|D^{l-1} f(0)\right\|^{2}+\int_{0}^{t}\left\|D^{l} f(\tau)\right\|^{2} d \tau\right\}$.

Corollary 4. In Proposition 1, we further suppose $f \equiv 0$.

Then there exist the positive constants $t_{0}$ and $\delta(<1)$ such that for $\left\|D^{s+1} u(0)\right\| \leq \delta e(42)$ has a unique solution

$$
u(x, t) \in \mathscr{D}\left(0, t_{0} \mid e\right) \cap \mathscr{E}_{t}^{0}\left(H^{s+1} \cap \stackrel{\circ}{H}^{s}\right) \cap \mathscr{E}_{t}^{i}\left(\stackrel{\circ}{H}^{s+1-i}\right) \quad(1 \leq i \leq s+1)
$$

where $t_{0}$ and $\delta$ depend on $e_{0}$ but not on $e$.

Proof of Proposition 1. Extending $v$ on $-1<t \leq 0$ properly, we have from Assumption 1 and (43) that

$$
\begin{array}{ll}
\tilde{a}_{i j}(x, t) \equiv a_{i j}(x, t, D v(x, t)) \in \mathscr{E}_{t}^{i}\left(H^{s-i}\right) & (0 \leq i \leq s) \\
\sum \tilde{a}_{i j}(x, t) \xi_{i} \xi_{j} \geq a_{1} \sum \xi_{i}^{2} & a_{1}>0 \\
\tilde{a}_{i j}(x, t)=\tilde{a}_{j i}(x, t) &
\end{array}
$$

for all $x \in \Omega, \xi \in R^{n}$ and $t>-1$. Therefore, taking care of regularity of $\tilde{a}_{i j}$, we have from the arguments in [4] that if $\phi \in H^{2} \cap \stackrel{H}{1}^{1}, \psi \in \dot{H}^{1}$ and $f \in \mathscr{E}_{t}^{1}\left(L^{2}\right)$ (42) has a unique solution $u(x, t)$ which satisfies

$$
\begin{aligned}
& u(x, t) \in \mathscr{E}_{t}^{o}\left(H^{2} \cap \stackrel{\circ}{H}^{1}\right) \cap \mathscr{E}_{t}^{1}\left(\stackrel{\circ}{H}^{1}\right) \cap \mathscr{E}_{t}^{2}\left(L^{2}\right) \\
& \left\|D^{2} u(t)\right\|^{2} \leq c \exp (c t)\left\{\left\|D^{2} u(0)\right\|^{2}+\|f(0)\|^{2}+\int_{0}^{t}\left\|D_{t}^{1} f(\tau)\right\|^{2} d \tau\right\}
\end{aligned}
$$

So let us show the regularity of the solution. Now we put

$$
w^{0}=u_{t}, \quad w^{k}=u_{k} \quad(1 \leq k \leq n) .
$$

Then differentiating (42), we have

$$
L_{v}\left(w^{k}\right)=-\sum_{i, j=1}^{n} a_{i j}^{k} w_{j}^{i}+f_{k} \quad \text { for } \quad 0 \leq k \leq n
$$

where $a_{i j}^{0}=\frac{\partial}{\partial t} a_{i j}, a_{i j}^{k}=\frac{\partial}{\partial x_{k}} a_{i j}(1 \leq k \leq n)$ and $f_{0}=f_{t}$. In order to solve the (46) we make the sequences $\left\{w^{k, m}\right\}(m \geq 0)$ as follows; for $m=0$

$$
w^{0,0} \equiv \psi, \quad w^{k, 0} \equiv \phi_{k} \quad(1 \leq k \leq n),
$$


for $m \geq 1$

$$
\begin{gathered}
L_{v}\left(w^{k, m}\right)=-\sum_{i, j=1}^{n} a_{i j}^{k} w_{j}^{i, m-1}+f_{k} \quad(0 \leq k \leq n) \\
\left\{\begin{array}{l}
w^{0, m}(0)=\psi \\
w_{t}^{0, m}(0)=-\sum a_{i j}^{0}(x, 0) \phi_{i j}+f(0)\left\{\begin{array}{l}
w^{k, m}(0)=\phi_{k} \\
w_{t}^{k, m}(0)=\psi_{k} \\
\left.w^{0, m}\right|_{\partial \Omega}=0,
\end{array} \quad(1 \leq k \leq n)\right.
\end{array}\right.
\end{gathered}
$$

Using the assumptions, we have from (45) that

$$
\begin{gathered}
w^{k, m}(x, t) \in \mathscr{E}_{i}^{0}\left(H^{2} \cap \stackrel{\circ}{H}^{1}\right) \cap \mathscr{E}_{t}^{1}\left(\dot{H}^{1}\right) \cap \mathscr{E}_{t}^{2}\left(L^{2}\right) \quad(m \geq 0,0 \leq k \leq n), \\
\sum_{k=0}^{n}\left\|D^{2}\left(w^{k, m+1}-w^{k, m}\right)(t)\right\|^{2} \leq c \int_{0}^{t}\left\|D_{t}^{1}\left(\sum_{\substack{k=0 \\
i, j=1}}^{n} a_{i j}^{k}\left(w^{k, m}-w^{k, m-1}\right)\right)(\tau)\right\|^{2} d \tau \\
\leq c \int_{0}^{t} \sum_{k=0}^{n}\left\|D^{2}\left(w^{k, m}-w^{k, m-1}\right)(\tau)\right\|^{2} d \tau, \\
\sum_{k=0}^{n}\left\|D^{2} w^{k, m}(t)\right\|^{2} \leq c \exp (c t)\left\{\sum_{k=0}^{n}\left\|D^{2} w^{k, m}(0)\right\|^{2}+\left\|D^{1} f(0)\right\|^{2}\right. \\
\left.+\int_{0}^{t}\left\{\sum_{k=0}^{n}\left\|D^{2} w^{k, m-1}(\tau)\right\|^{2}+\left\|D^{2} f(\tau)\right\|^{2}\right\} d \tau\right\} \quad(m \geq 1) .
\end{gathered}
$$

From (47) we can get the solutions of (46). So (42) has a solution

$$
u(x, t) \in \mathscr{E}_{t}^{0}\left(H^{3} \cap \stackrel{\circ}{H}^{2}\right) \cap \mathscr{E}_{t}^{i+1}\left(\stackrel{\circ}{H}^{2-i}\right) \quad(0 \leq i \leq 2) .
$$

Furthermore (45) and (47) give

$$
\left\|D^{3} u(t)\right\|^{2} \leq c \exp (c t)\left\{\left\|D^{3} u(0)\right\|^{2}+\left\|D^{1} f(0)\right\|^{2}+\int_{0}^{t}\left\|D^{2} f(\tau)\right\|^{2} d \tau\right\} .
$$

Similarly we can get the regularity up to the order $s+1$ and the energy inequality (44) step by step.

Q.E.D.

Proof of Corollary 4. It follows from (44) that

$$
\left\|D^{s+1} u(t)\right\|^{2} \leq c \exp (c t)\left\|D^{s+1} u(0)\right\|^{2}
$$

where $c$ depends on $e_{0}, h_{0}$ and $a_{1}$ but not on $e$. Therefore choosing $t_{0}$ 
and $\delta$ such that

$$
\exp \left(c t_{0}\right) \leq 2, \quad 2 c \delta^{2} \leq 1
$$

we have

$$
\sup _{0 \leq t \leq t_{0}}\left\|D^{s+1} u(t)\right\| \leq e
$$

Q.E.D.

Under the above preparations, we have the following

Theorem 4. We suppose Assumption 1. Moreover we suppose that $\phi \in H^{s+1} \cap \stackrel{\circ}{H}^{s}$ and $\psi \in \stackrel{\circ}{H}^{s}$ satisfy the compatibility condition. Then there exist the positive constants $t_{0}$ and $\delta(<1)$ such that if $\left\|D^{s+1} u(0)\right\|$ $\leq \delta e\left(0<e \leq e_{0}\right)$, then the initial-boundary problem for (41) has a unique solution $u(x, t)$ which satisfies

$$
u(x, t) \in \mathscr{D}\left(0, t_{0} \mid e\right) \quad \text { and } \quad D^{k, l} u(x, t) \in \mathscr{E}_{t}^{\circ}\left(\stackrel{\circ}{H}^{1}\right) \quad(0 \leq k+l \leq s, k \neq s) .
$$

Proof. We first note that we can construct some $w(x, t) \in \mathscr{E}_{t}^{i}\left(H^{s+1-i}\right)$ $(0 \leq i \leq s+1)$ satisfying

$$
D^{s+1} w(0)=D^{s+1} u(0) \quad \text { and } \quad \delta\left\|D^{s+1} w(t)\right\| \leq\left\|D^{s+1} w(0)\right\| \quad\left(0 \leq t \leq t_{0}\right) .
$$

Then we construct the approximate sequence as follows;

$$
\begin{aligned}
& u^{0}=w, \\
& L_{u^{m-1}}\left(u^{m}\right)=0 \quad(m \geq 1) \\
& \left\{\begin{array}{l}
u^{m}(0)=\phi \\
u_{t}^{m}(0)=\psi \\
\left.u^{m}\right|_{\partial \Omega}=0 .
\end{array}\right.
\end{aligned}
$$

Since $D^{s+1} u^{m}(0)=D^{s+1} w(0)$ for all $m \geq 0$, it follows by Corollary 4 that

$$
u^{m}(x, t) \in \mathscr{D}\left(0, t_{0} \mid e\right) \cap \mathscr{E}_{t}^{0}\left(H^{s+1} \cap \stackrel{\circ}{H}^{s}\right) \cap \mathscr{E}_{t}^{i}\left(\stackrel{\circ}{H}^{s+1-i}\right) \quad(1 \leq i \leq s+1)
$$

for all $m \geq 0$. From (49) we have 


$$
L_{u^{m}}\left(u^{m+1}-u^{m}\right)=A^{m}\left(u^{m}-u^{m-1}\right)
$$

where $A^{m}\left(u^{m}-u^{m-1}\right)=\sum\left\{a_{i j}\left(x, t, D u^{m}\right)-a_{i j}\left(x, t, D u^{m-1}\right)\right\} u_{i j}^{m}$. So from (44) we have

$$
\left\|D^{s}\left(u^{m+1}-u^{m}\right)(t)\right\|^{2} \leq c \int_{0}^{t}\left\|D^{s-1} A^{m}\left(u^{m}-u^{m-1}\right)(\tau)\right\|^{2} d \tau
$$

On the other hand we have from iv) of Lemma 4

$$
\left\|D^{s-1} A^{m}\left(u^{m}-u^{m-1}\right)\right\|^{2} \leq c\left(e_{0}\right)\left\|D^{s}\left(u^{m}-u^{m-1}\right)\right\|^{2} .
$$

Substituting (52) to (51), we have

$$
\left\|D^{s}\left(u^{m+1}-u^{m}\right)(t)\right\|^{2} \leq c \int_{0}^{t}\left\|D^{s}\left(u^{m}-u^{m-1}\right)(\tau)\right\|^{2} d \tau \quad \text { for all } \quad m \geq 1
$$

Therefore (50) and (53) give a solution of (41) satisfying

$$
D^{s+1} u(x, t) \in L_{t}^{\infty}\left(L^{2}\right) \quad \text { and } \quad D^{k, l} u(x, t) \in L_{t}^{\infty}\left(\stackrel{\circ}{H}^{1}\right) \quad(0 \leq k+l \leq s, k \neq s) .
$$

Finally the uniqueness follows from the similar energy inequality as (53) and the regularity $D^{s+1} u \in \mathscr{E}_{t}^{0}\left(L^{2}\right)$ follows from

$$
\sup _{0 \leq t \leq t_{0}}\left\|D^{s+1}\left(\varphi_{\delta} * u-\varphi_{\delta^{\prime}} * u\right)(t)\right\| \rightarrow 0, \quad \text { as } \quad \delta, \delta^{\prime} \rightarrow 0
$$

where $\varphi_{\delta} *$ is the mollifier with respect to $t$ and $u$ is extended properly on $-\varepsilon<t<t_{0}+\varepsilon$.

Q.E.D.

\section{Acknowledgment}

The author would like to thank Prof. Yujiro Ohya and Dr. Takaaki Nishida for their many valuable comments and suggestions.

\section{References}

[1] Dionne, P., Sur les problème de Cauchy hyperboliques bien posés, J. Analyse Math., 10 (1962), 1-90.

[2] Matsumura, A., On the asymptotic behavior of solutions of semilinear wave equations, Publ. RIMS, Kyoto Univ., 12 (1976), 169-189.

[3] Mizohata, S., Theory of partial differential equations, Cambridge UP., 1973.

[4] — Quelque problèmes au bord, du type mixte, pour des équations 
hyperboliques. Séminaire sur les équations aux derivées partielles, Collège de France, (1966-1967), 23-60.

[5] Nirenberg, L., On elliptic partial differential equations, Ann. Scuola Norm. Sup. Pisa., 13 (1959), 115-162.

[6] Nishida, T., Global smooth solutions for the second-order quasilinear wave equations with the first-order dissipation (to appear).

[7] Sattinger, D., Stability of nonlinear hyperbolic equations, Arch. Rational Mech. Anal., 28 (1968), 226-244.

[8] Rabinowitz, P., Periodic solutions of nonlinear partial differential equations, Comm. Pure Appl. Math., 20 (1967), 145-205.

[9] — Periodic solutions of nonlinear partial differential equations II, Comm. Pure Appl. Math., 22 (1969), 15-39. 
